\title{
Common fixed point theorems for generalized $k$-ordered contractions and $B$-contractions on noncommutative Banach spaces
}

\section{Qiaoling Xin and Lining Jiang*}

"Correspondence:

jianglining@bit.edu.cn

School of Mathematics and

Statistics, Beijing Institute of

Technology, Beijing, 100081, China

\section{算 Springer}

\begin{abstract}
The paper introduces the concepts of the generalized $k$-ordered contraction and the $k$-ordered $B$-contraction in noncommutative Banach spaces. Then some common fixed point theorems are given. As an application, the existence and uniqueness theorem for a common solution of integral equations is presented.
\end{abstract}

MSC: $47 \mathrm{H} 10$

Keywords: common fixed point; noncommutative Banach space; generalized k-ordered contraction; $k$-ordered $B$-contraction

\section{Introduction}

The study of common fixed points of mappings satisfying certain contractive conditions has many applications and has been at the center of vigorous research activity. In 1976, Jungck [1] proved common fixed point theorems for commuting mappings in metric spaces, generalizing the Banach contraction principle. Later, Das and Naik [2] investigated the corresponding common fixed point result for Ćirić's fixed point theorem [3]. Thereafter the concept of commuting mappings has been developed in various directions. One of such notions which is weaker than commuting is the concept of compatibility introduced by Jungck [4]. In common fixed point problems this concept and its generalizations have been used extensively (see [5-7]), e.g., Ćirić et al. [7] proved some common fixed point theorems for two pairs of weakly compatible mappings satisfying a generalized contraction condition on partial metric spaces. For a survey of common fixed point theory, its applications, comparison of different contractive conditions, and related results, one can refer to [8-10] and references contained therein.

Recently, Huang and Zhang [11] generalized the notion of metric spaces by substituting the set of real numbers with the ordered Banach space, and defined the concept of cone metric spaces. Based on the notion of cone metric spaces, several fixed point theorems were obtained for mappings satisfying certain contractive type condition; see for example [12-18]. Subsequently, Xin and Jiang [19] introduced noncommutative Banach spaces which generalize the concept of Banach spaces and established fixed point theorems for mappings with the $k$-ordered contractive condition.

(c) 2015 Xin and Jiang. This article is distributed under the terms of the Creative Commons Attribution 4.0 International License (http://creativecommons.org/licenses/by/4.0/), which permits unrestricted use, distribution, and reproduction in any medium, provided you give appropriate credit to the original author(s) and the source, provide a link to the Creative Commons license, and indicate if changes were made. 
The paper will give some common fixed theorems in the framework of noncommutative Banach spaces. In details, we firstly introduce the concept of generalized $k$-ordered contractions in noncommutative Banach spaces, and prove some common fixed point theorems for generalized $k$-ordered contractions which generalize the results in [19] (see Theorem 2.1). Then the notion of $k$-ordered $B$-contractions is introduced in noncommutative Banach spaces and the corresponding common fixed point theorems are given. At the end, to see the applicability of our results, we give an existence and uniqueness theorem for a common solution of integral equations.

Now we recall the definition of noncommutative Banach spaces and some of their properties which will be needed in the sequel [19].

Definition 1.1 Let $E$ be a group with a unit $e$ and suppose that there exists a metric $d$ on $E$ such that $(E, d)$ is a complete metric space. $E$ is said to be a noncommutative Banach space if the following conditions hold:

(1) for any $x, y, z \in E$, we have $d(x z, y z)=d(x, y)$;

(2) there exists a binary continuous operation

$$
F: \mathbb{R} \times E \rightarrow E, \quad(\alpha, x) \mapsto x^{\alpha}
$$

such that $F(-1, x)=x^{-1}$ is exactly the inverse of $x$ in the group $E$ and $F(0, x)=x^{0}=e$ is the unit in the group $E$, and that

$$
F(m n, x)=F(m, F(n, x)), \quad F(m+n, x)=F(m, x) F(n, x)
$$

for $m, n \in \mathbb{R}, x \in E$;

(3) for any $x \in E$, there exists a constant $M_{x}>0$ such that

$$
d\left(x^{\alpha}, e\right) \leq M_{x}|\alpha|, \quad \forall \alpha \in \mathbb{R} .
$$

It can be shown that all Banach spaces and unitary groups of Hilbert spaces are all noncommutative Banach spaces. For more details, one can refer to [19].

Let $E$ be a noncommutative Banach space. A subset $P$ of $E$ is called a cone if and only if:

(1) $P$ is nonempty, closed, and $P \neq\{e\}$;

(2) $x, y \in P$ and $\alpha, \beta \in \mathbb{R}^{+}$imply $x^{\alpha} y^{\beta} \in P$;

(3) $P \cap P^{-1}=\{e\}$ where $P^{-1}=\left\{x^{-1}: x \in P\right\}$.

Given a cone $P \subseteq E$, we define a partial ordering $\lesssim$ in $E$ with respect to $P$ by $x \lesssim y$ if and only if $y^{\beta} x^{-\beta} \in P$ for all $\beta \in[0,1]$. A cone $P$ is said to be normal if there is a number $N>0$ such that for all $x, y \in E$,

$$
e \lesssim x \lesssim y \quad \Rightarrow \quad d(x, e) \leq N d(y, e)
$$

The least positive number $N$ satisfying above is called the normal constant of $P$.

For $x, y \in E$, if either $x \lesssim y$ or $y \lesssim x$ holds, we say $x$ and $y$ are comparable and denoted by

$$
\vee(x, y)= \begin{cases}x, & y \lesssim x \\ y, & x \lesssim y .\end{cases}
$$


Lemma 1.1 Suppose that $P$ is a cone in E. For $x, y \in E$,

(1) set $x \lesssim y$, then $x^{\alpha} \lesssim y^{\alpha}$ holds for all $\alpha \in[0,1]$;

(2) if $x \in P$ and there exists $\lambda \in[0,1)$ such that $x \lesssim x^{\lambda}$, then $x=e$;

(3) if $x$ and $y$ are comparable, then $x y^{-1}$ and $y x^{-1}$ are comparable, and $e \lesssim \vee\left(x y^{-1}, y x^{-1}\right)$;

(4) if $x$ and $y$ are comparable, then $d\left(\vee\left(x y^{-1}, y x^{-1}\right), e\right)=d(x, y)$.

Proof (1) Let $x \lesssim y$, we have $y^{\beta} x^{-\beta} \in P$ for all $\beta \in[0,1]$. Since $\alpha \beta \in[0,1]$ for any $\alpha \in[0,1]$, we see $\left(y^{\alpha}\right)^{\beta}\left(x^{\alpha}\right)^{-\beta}=y^{\alpha \beta} x^{-\alpha \beta} \in P$, which means $x^{\alpha} \lesssim y^{\alpha}$.

(2) From $x \lesssim x^{\lambda}$, we know $x^{\lambda-1} \in P$. It follows from $\frac{1}{1-\lambda}>0$ and the definition of cone that $x^{-1} \in P$, which together with $x \in P$ yields $x=e$.

(3) One may suppose that $x \lesssim y$, which means $y x^{-1} \in P$. For all $\beta \in[0,1],\left(y x^{-1}\right)^{\beta}\left(x y^{-1}\right)^{-\beta}=$ $\left(y x^{-1}\right)^{\beta}\left(\left(y x^{-1}\right)^{-1}\right)^{-\beta}=\left(y x^{-1}\right)^{2 \beta} \in P$, then $x y^{-1} \lesssim y x^{-1}$. Furthermore $\left(y x^{-1}\right)^{\beta} e^{-\beta}=\left(y x^{-1}\right)^{\beta} \in P$, which implies $e \lesssim y x^{-1}$, and therefore $e \lesssim \vee\left(x y^{-1}, y x^{-1}\right)$.

(4) Assume that $x \lesssim y$, then $\vee\left(x y^{-1}, y x^{-1}\right)=y x^{-1}$. It follows immediately from Definition 1.1 that

$$
d\left(\vee\left(x y^{-1}, y x^{-1}\right), e\right)=d\left(y x^{-1}, e\right)=d(y, x)=d(x, y) .
$$

In the rest of the paper, we always suppose that $E$ is a noncommutative Banach space, $P$ is a normal cone in $E$ with the normal constant $N$ and $\lesssim$ is a partial ordering with respect to $P$.

\section{Common fixed point theorems for the generalized $\boldsymbol{k}$-ordered contraction}

In this section, we give the generalized $k$-ordered contraction in noncommutative Banach spaces, and prove some common fixed point theorems for the generalized $k$-ordered contraction which generalize the results in [19].

Definition 2.1 Let $A, B: E \rightarrow E$ be two mappings. We say that the mapping $A$ is the generalized $k$-ordered contraction if there exists $k \in(0,1)$ such that for all $x, y \in E$, if $x$ and $y$ are comparable, then $A x$ and $B y$ are comparable, and, moreover,

$$
\vee\left(A x(B y)^{-1}, B y(A x)^{-1}\right) \lesssim \vee\left(x y^{-1}, y x^{-1}\right)^{k} .
$$

In particular, if $A=B, A$ is called the $k$-ordered contraction, which can also be found in [19].

Theorem 2.1 Let $A, B: E \rightarrow E$ be two mappings satisfying the following conditions:

(i) $A$ or $B$ is continuous;

(ii) $A$ is the generalized $k$-ordered contraction;

(iii) there is $x_{0} \in E$ such that $x_{0}$ and $A x_{0}$ are comparable.

Then $A$ and $B$ have a common fixed point in $E$, that is, there exists $x^{*} \in E$ such that $A x^{*}=$ $B x^{*}=x^{*}$.

Proof Starting with the given $x_{0}$, construct a sequence $\left\{x_{n}\right\}_{n=0}^{\infty}$ by $x_{2 n+1}=A x_{2 n}$ and $x_{2 n+2}=$ $B x_{2 n+1}$ for all $n \in \mathbb{N}$. Using the condition (ii), it can easily be shown that $x_{n}$ and $x_{n+1}$ are comparable. In particular, $x_{2 n+1}$ and $x_{2 n+2}$ are comparable. From the condition (ii) it follows 
that

$$
\begin{aligned}
\vee\left(x_{2 n+1} x_{2 n+2}^{-1}, x_{2 n+2} x_{2 n+1}^{-1}\right) & =\vee\left(A x_{2 n}\left(B x_{2 n+1}\right)^{-1}, B x_{2 n+1}\left(A x_{2 n}\right)^{-1}\right) \\
& \lesssim \vee\left(x_{2 n} x_{2 n+1}^{-1}, x_{2 n+1} x_{2 n}^{-1}\right)^{k}
\end{aligned}
$$

In a similar way one obtains

$$
\vee\left(x_{2 n} x_{2 n+1}^{-1}, x_{2 n+1} x_{2 n}^{-1}\right) \lesssim \vee\left(x_{2 n-1} x_{2 n}^{-1}, x_{2 n} x_{2 n-1}^{-1}\right)^{k}
$$

Now, by induction, we can get

$$
\vee\left(x_{n} x_{n+1}^{-1}, x_{n+1} x_{n}^{-1}\right) \lesssim \vee\left(x_{0} x_{1}^{-1}, x_{1} x_{0}^{-1}\right)^{k^{n}}
$$

Since $P$ is a normal cone with the normal constant $N$,

$$
d\left(\vee\left(x_{n} x_{n+1}^{-1}, x_{n+1} x_{n}^{-1}\right), e\right) \leq N d\left(\vee\left(x_{0} x_{1}^{-1}, x_{1} x_{0}^{-1}\right)^{k^{n}}, e\right)
$$

Using Definition 1.1 and Lemma 1.1(4), we conclude that

$$
d\left(x_{n}, x_{n+1}\right) \leq k^{n} N M_{x_{0} x_{1}^{-1}}
$$

As a result, $\left\{x_{n}\right\}_{n=0}^{\infty}$ is a Cauchy sequence in $E$. Since $E$ is a complete metric space, there exists a point $x^{*} \in E$ such that $x_{n} \rightarrow x^{*}$ as $n \rightarrow \infty$.

Suppose that $A$ is continuous, one has $x^{*}=\lim _{n \rightarrow \infty} A x_{2 n}=A x^{*}$. This shows that $x^{*}$ is a fixed point of $A$. Now, by the reflexivity of the partial ordering $\lesssim$ in $E$, we know $x^{*} \lesssim x^{*}$. The condition (ii) implies that $A x^{*}$ and $B x^{*}$ are comparable, and

$$
\vee\left(x^{*}\left(B x^{*}\right)^{-1}, B x^{*}\left(x^{*}\right)^{-1}\right)=\vee\left(A x^{*}\left(B x^{*}\right)^{-1}, B x^{*}\left(A x^{*}\right)^{-1}\right) \lesssim e^{k}=e .
$$

By the definition of the partial ordering in $E$, we have

$$
\vee\left(x^{*}\left(B x^{*}\right)^{-1}, B x^{*}\left(x^{*}\right)^{-1}\right)^{-1} \in P .
$$

Again, $x^{*}=A x^{*}$ and $B x^{*}$ are comparable, then by Lemma 1.1(3), we know $e \lesssim \vee\left(x^{*}\left(B x^{*}\right)^{-1}\right.$, $\left.B x^{*}\left(x^{*}\right)^{-1}\right)$. That is

$$
\vee\left(x^{*}\left(B x^{*}\right)^{-1}, B x^{*}\left(x^{*}\right)^{-1}\right) \in P
$$

Hence

$$
\vee\left(x^{*}\left(B x^{*}\right)^{-1}, B x^{*}\left(x^{*}\right)^{-1}\right) \in P \cap P^{-1}=\{e\},
$$

and then $B x^{*}=x^{*}$.

Similarly, if $B$ is continuous, again we have $A x^{*}=x^{*}$. Therefore, $A$ and $B$ have a common fixed point.

The following theorem gives the sufficient condition for the uniqueness of a common fixed point of $A$ and $B$ in Theorem 2.1. 
Theorem 2.2 In addition to the hypotheses of Theorem 2.1, assume that for all $x, y \in E$, there exists $w \in E$ depending on $x$ and $y$ such that $w$ is comparable with $x$ and $y$. Then $A$ and $B$ have a unique common fixed point.

Proof The set of common fixed points of $A$ and $B$ is not empty due to Theorem 2.1; suppose now that $x$ and $y$ are two common fixed points of $A$ and $B$, i.e., $A x=B x=x$, $A y=B y=y$. We distinguish two cases:

Case 1. If $x$ and $y$ are comparable, that is, $A x$ and $B y$ are comparable, then by the condition (ii), we obtain

$$
\begin{aligned}
\vee\left(x y^{-1}, y x^{-1}\right) & =\vee\left(A x(B y)^{-1}, B y(A x)^{-1}\right) \\
& \lesssim \vee\left(x y^{-1}, y x^{-1}\right)^{k},
\end{aligned}
$$

which shows $\vee\left(x y^{-1}, y x^{-1}\right)^{k-1} \in P$. According to Lemma 1.1(2), $\vee\left(x y^{-1}, y x^{-1}\right)=e$ and this gives us $x=y$.

Case 2. If $x$ is not comparable with $y$, then there exists $w \in E$ which is comparable with $x$ and $y$. The condition (ii) implies that $B^{n} w$ is comparable with $A^{n} x=x$ and $A^{n} y=y$ for $n=0,1,2, \ldots$. Moreover,

$$
\begin{aligned}
\vee\left(x\left(B^{n} w\right)^{-1}, B^{n} w x^{-1}\right) & =\vee\left(A^{n} x\left(B^{n} w\right)^{-1}, B^{n} w\left(A^{n} x\right)^{-1}\right) \\
& \lesssim \vee\left(A^{n-1} x\left(B^{n-1} w\right)^{-1}, B^{n-1} w\left(A^{n-1} x\right)^{-1}\right)^{k} \\
& =\vee\left(x\left(B^{n-1} w\right)^{-1}, B^{n-1} w x^{-1}\right)^{k} .
\end{aligned}
$$

By induction, we conclude that

$$
\vee\left(x\left(B^{n} w\right)^{-1}, B^{n} w x^{-1}\right) \lesssim \vee\left(x w^{-1}, w x^{-1}\right)^{k^{n}} .
$$

Then

$$
d\left(x, B^{n} w\right) \leq k^{n} N M_{x w^{-1}} .
$$

Analogously, it can be proved that

$$
d\left(y, B^{n} w\right) \leq k^{n} N M_{y w^{-1}}
$$

Using the triangular inequality, one can obtain

$$
\begin{aligned}
d(x, y) & \leq d\left(x, B^{n} w\right)+d\left(B^{n} w, y\right) \\
& \leq k^{n} N\left(M_{x w^{-1}}+M_{y w^{-1}}\right) \rightarrow 0 .
\end{aligned}
$$

Hence $x=y$, which is a contradiction. Consequently, $x$ and $y$ are comparable, and from Case 1 , we know $x=y$.

Corollary 2.1 Let $A: E \rightarrow E$ be a continuous mapping satisfying the following conditions: 
(i) there exists $k \in(0,1)$ such that for all $x, y \in E$, if $x$ and $y$ are comparable, then $x$ and $A^{p} y, A x$ and Ay are comparable, respectively, and, moreover,

$$
\vee\left(A^{n} x\left(A^{m} y\right)^{-1}, A^{m} y\left(A^{n} x\right)^{-1}\right) \lesssim \vee\left(x y^{-1}, y x^{-1}\right)^{k}
$$

for any $n, m, p \in \mathbb{N}$;

(ii) there is $x_{0} \in E$ such that $x_{0}$ and $A x_{0}$ are comparable;

(iii) for all $x, y \in E$, there exists $w \in E$ such that $w$ is comparable with $x$ and $y$.

Then $A$ has a unique fixed point in $E$.

Proof It follows from Theorem 2.1 and Theorem 2.2 by putting $A^{n} \equiv A$ and $A^{m} \equiv B$.

As a consequence of the previous corollary, we obtain a fixed point theorem for the $k$-ordered contraction on a noncommutative Banach space, which can also be seen in [19].

Corollary 2.2 Let $A: E \rightarrow E$ be a continuous mapping satisfying the following conditions:

(i) there exists $k \in(0,1)$ such that for all $x, y \in E$, if $x$ and $y$ are comparable, then $A x$ and Ay are comparable, and, moreover,

$$
\vee\left(A x(A y)^{-1}, A y(A x)^{-1}\right) \lesssim \vee\left(x y^{-1}, y x^{-1}\right)^{k} ;
$$

(ii) there is $x_{0} \in E$ such that $x_{0}$ and $A x_{0}$ are comparable;

(iii) for all $x, y \in E$, there exists $w \in E$ such that $w$ is comparable with $x$ and $y$.

Then $A$ has a unique fixed point in $E$.

\section{Common fixed point theorems for the $k$-ordered $B$-contraction}

In the following we shall introduce the notion of $k$-ordered $B$-contractions in the framework of noncommutative Banach spaces and prove some common fixed point theorems. Let us start with the following definition.

Definition 3.1 Let $A, B: E \rightarrow E$ be two mappings. We say that the mapping $A$ is the $k$-ordered $B$-contraction if there exists $k \in(0,1)$ such that for each $x, y \in E$ satisfying $B x$ and $B y$ are comparable, $A x$ and $A y$ are comparable and

$$
\vee\left(A x(A y)^{-1}, A y(A x)^{-1}\right) \lesssim \vee\left(B x(B y)^{-1}, B y(B x)^{-1}\right)^{k} .
$$

Theorem 3.1 Let $A, B: E \rightarrow E$ be two continuous mappings such that $R(A) \subseteq R(B)$. Suppose that the following conditions hold:

(i) $A$ and $B$ commute;

(ii) $A$ is the $k$-ordered B-contraction;

(iii) there exists $x_{0} \in E$ such that $A x_{0}$ and $B x_{0}$ are comparable;

(iv) if $\left\{B x_{n}\right\}_{n=0}^{\infty}$ is a sequence in $E$ which has comparable adjacent terms and converges to some $z$ in $E$, then $B z$ and $B(B z)$ are comparable.

Then $A$ and $B$ have a common fixed point in $E$.

Proof Let $x_{0} \in E$ be such that $A x_{0}$ and $B x_{0}$ are comparable and $x_{1} \in E$ be chosen such that $B x_{1}=A x_{0}$. This can be done since $R(A) \subseteq R(B)$. Let $x_{2} \in E$ be such that $B x_{2}=A x_{1}$. 
Continuing this process, we can construct a sequence $\left\{B x_{n}\right\}_{n=0}^{\infty}$ in $E$ such that $B x_{n+1}=A x_{n}$ for all $n \in \mathbb{N}$.

Since $A x_{0}$ and $B x_{0}$ are comparable and $B x_{1}=A x_{0}$, we find that $B x_{1}$ and $B x_{0}$ are comparable. Then by the condition (ii), $A x_{1}$ and $A x_{0}$ are comparable. Thus $B x_{2}$ and $B x_{1}$ are comparable. Continuing this process, we obtain $B x_{1}$ and $B x_{0}, B x_{2}$ and $B x_{1}, \ldots, B x_{n+1}$ and $B x_{n}$ are comparable, respectively. Notice that

$$
\begin{aligned}
\vee\left(B x_{n}\left(B x_{n+1}\right)^{-1}, B x_{n+1}\left(B x_{n}\right)^{-1}\right) & =\vee\left(A x_{n-1}\left(A x_{n}\right)^{-1}, A x_{n}\left(A x_{n-1}\right)^{-1}\right) \\
& \lesssim \vee\left(B x_{n-1}\left(B x_{n}\right)^{-1}, B x_{n}\left(B x_{n-1}\right)^{-1}\right)^{k}
\end{aligned}
$$

Inductively the following holds:

$$
\vee\left(B x_{1}\left(B x_{2}\right)^{-1}, B x_{2}\left(B x_{1}\right)^{-1}\right) \lesssim \vee\left(B x_{0}\left(B x_{1}\right)^{-1}, B x_{1}\left(B x_{0}\right)^{-1}\right)^{k}
$$

By the above and Lemma 1.1(1), we obtain

$$
\vee\left(B x_{n}\left(B x_{n+1}\right)^{-1}, B x_{n+1}\left(B x_{n}\right)^{-1}\right) \lesssim \vee\left(B x_{0}\left(B x_{1}\right)^{-1}, B x_{1}\left(B x_{0}\right)^{-1}\right)^{k^{n}} .
$$

Following an argument similar to that given in Theorem 2.1, we find that $\left\{B x_{n}\right\}_{n=0}^{\infty}$ is a Cauchy sequence, and there is some $p \in E$ such that $A x_{n-1}=B x_{n} \rightarrow p$ as $n \rightarrow \infty$.

Again, since $A, B$ are continuous and commute, we get

$$
A p=A\left(\lim _{n \rightarrow \infty} B x_{n}\right)=\lim _{n \rightarrow \infty} A B x_{n}=\lim _{n \rightarrow \infty} B A x_{n}=B\left(\lim _{n \rightarrow \infty} A x_{n}\right)=B p .
$$

Let $w=A p=B p$, then

$$
A w=A(B p)=B(A p)=B w .
$$

From the condition (iv), we know $w$ and $A w$ are comparable. Since $A$ is the $k$-ordered $B$-contraction, we have

$$
\begin{aligned}
\vee\left(w(A w)^{-1}, A w w^{-1}\right) & =\vee\left(A p(A w)^{-1}, A w(A p)^{-1}\right) \\
& \lesssim \vee\left(B p(B w)^{-1}, B w(B p)^{-1}\right)^{k} \\
& =\vee\left(w(A w)^{-1}, A w w^{-1}\right)^{k}
\end{aligned}
$$

which shows $\vee\left(w(A w)^{-1}, A w w^{-1}\right)^{k-1} \in P$. Using Lemma 1.1(2), it follows that $w=A w=B w$. Therefore, $A$ and $B$ have a common fixed point.

In addition to the hypotheses of Theorem 3.1, suppose that for all $x, y \in E$, there exists $z \in E$ depending on $x$ and $y$ such that $B z$ is comparable with $B x$ and $B y$. Then $A$ and $B$ have a unique common fixed point. Indeed, assume that there exist $x, y \in E$ which are two common fixed points of $A$ and $B$. We claim that $x=y$.

By assumption, there exists $z \in E$ such that $B z$ is comparable with $B x$ and $B y$. Define a sequence $\left\{B z_{n}\right\}_{n=0}^{\infty}$ such that $z_{0}=z$ and $B z_{n}=A z_{n-1}$ for all $n$. 
Further, set $x_{0}=x$ and $y_{0}=y$, and in the same way define $\left\{B x_{n}\right\}_{n=0}^{\infty}$ and $\left\{B y_{n}\right\}_{n=0}^{\infty}$ such that $B x_{n}=A x_{n-1}, B y_{n}=A y_{n-1}$ for all $n \in \mathbb{N}$. Since $B x$ is comparable with $B z$ and $A$ is the $k$-ordered $B$-contraction, we find that $x$ and $A z$ are comparable. Again, since $x=B x$ and $A z=B z_{1}, x$ and $A z_{1}$ are comparable. Recursively, $x$ and $A z_{n}$ are comparable. By the condition (ii), we have

$$
\begin{aligned}
\vee\left(x\left(A z_{n}\right)^{-1}, A z_{n} x^{-1}\right) & \lesssim \vee\left(x\left(A z_{n-1}\right)^{-1}, A z_{n-1} x^{-1}\right)^{k} \\
& \lesssim \cdots \\
& \lesssim \vee\left(x(A z)^{-1}, A z x^{-1}\right)^{k^{n}} .
\end{aligned}
$$

The same reasoning as that in Theorem 2.2 tells us that $x=y$, which means that $x$ is the unique common fixed point of $A$ and $B$.

Theorem 3.2 Let $A, B: E \rightarrow E$ be two continuous mappings such that $R(A) \subseteq R(B)$. Suppose that the following conditions hold:

(i) A and B commute;

(ii) there exist nonnegative real numbers $s, k$, and $h$ with $s>\max \{k+h, 1+h\}$ such that for all $\beta \in[0,1]$, if $x$ and $y$ are comparable, then $A x$ and $A y, A x$, and $B x$, Ay and By are comparable, respectively, and furthermore

$$
\vee\left(A x(A y)^{-1}, A y(A x)^{-1}\right)^{s \beta} \lesssim \vee\left(A x(B x)^{-1}, B x(A x)^{-1}\right)^{k \beta} \vee\left(A y(B y)^{-1}, B y(A y)^{-1}\right)^{h \beta} ;
$$

(iii) if $\left\{B x_{n}\right\}_{n=0}^{\infty}$ is a sequence in $E$ which has comparable adjacent terms and converges to some $z$ in $E$, then $B z$ and $B(B z)$ are comparable.

Then $A$ and $B$ have a common fixed point in $E$.

Proof Let $x_{0}$ be an arbitrary point in $E$. By the reflexivity of the partial ordering ' $\lesssim$ ' in $E$, $x_{0} \lesssim x_{0}$, which together with the condition (ii) shows that $A x_{0}$ and $B x_{0}$ are comparable. Following the lines of the proof of Theorem 3.1, we can construct a sequence $\left\{B x_{n}\right\}_{n=0}^{\infty}$ with $B x_{n+1}=A x_{n}$ for all $n \in \mathbb{N}$. Then from the condition (ii), we can obtain

$$
\begin{aligned}
\vee & \left(B x_{n}\left(B x_{n+1}\right)^{-1}, B x_{n+1}\left(B x_{n}\right)^{-1}\right)^{s \beta} \\
& =\vee\left(A x_{n-1}\left(A x_{n}\right)^{-1}, A x_{n}\left(A x_{n-1}\right)^{-1}\right)^{s \beta} \\
& \lesssim \vee\left(A x_{n-1}\left(B x_{n-1}\right)^{-1}, B x_{n-1}\left(A x_{n-1}\right)^{-1}\right)^{k \beta} \vee\left(A x_{n}\left(B x_{n}\right)^{-1}, B x_{n}\left(A x_{n}\right)^{-1}\right)^{h \beta} \\
& =\vee\left(B x_{n}\left(B x_{n-1}\right)^{-1}, B x_{n-1}\left(B x_{n}\right)^{-1}\right)^{k \beta} \vee\left(B x_{n+1}\left(B x_{n}\right)^{-1}, B x_{n}\left(B x_{n+1}\right)^{-1}\right)^{h \beta} .
\end{aligned}
$$

By the definition of the partial ordering in $E$ with respect to $P$, one can get

$$
\vee\left(B x_{n}\left(B x_{n-1}\right)^{-1}, B x_{n-1}\left(B x_{n}\right)^{-1}\right)^{k \beta} \vee\left(B x_{n+1}\left(B x_{n}\right)^{-1}, B x_{n}\left(B x_{n+1}\right)^{-1}\right)^{-(s-h) \beta} \in P .
$$

That is,

$$
\vee\left(B x_{n+1}\left(B x_{n}\right)^{-1}, B x_{n}\left(B x_{n+1}\right)^{-1}\right)^{s-h} \lesssim \vee\left(B x_{n}\left(B x_{n-1}\right)^{-1}, B x_{n-1}\left(B x_{n}\right)^{-1}\right)^{k}
$$


And from Lemma 1.1(1), one can obtain

$$
\vee\left(B x_{n+1}\left(B x_{n}\right)^{-1}, B x_{n}\left(B x_{n+1}\right)^{-1}\right) \lesssim \vee\left(B x_{n}\left(B x_{n-1}\right)^{-1}, B x_{n-1}\left(B x_{n}\right)^{-1}\right)^{\frac{k}{s-h}}
$$

The same reasoning that in Theorem 2.1 gives us that $\left\{B x_{n}\right\}_{n=0}^{\infty}$ is a Cauchy sequence, and consequently $\left\{B x_{n}\right\}_{n=0}^{\infty}$ is convergent to some $q \in E$. Also $A q=B q$.

Set $u=A q=B q$. Since $A$ and $B$ commute, $A u=A(B q)=B(A q)=B u$. We will show that $u=A u=B u$. In fact, from the conditions (ii) and (iii), we have

$$
\begin{aligned}
\vee\left(u(B u)^{-1}, B u u^{-1}\right) & =\vee\left(A q(A u)^{-1}, A u(A q)^{-1}\right) \\
& \lesssim \vee\left(A q(B q)^{-1}, B q(A q)^{-1}\right)^{k} \vee\left(A u(B u)^{-1}, B u(A u)^{-1}\right)^{h} \\
& =e .
\end{aligned}
$$

Analogous to the proof of Theorem 2.1, it is easily seen that $u=A u=B u$.

Remark 3.1 Let the conditions of Theorem 3.2 be satisfied, except that (ii) is replaced by

(ii') there exist nonnegative real numbers $s, k, h$, and $l$ with $s>\max \{k+h+l, 1+l\}$ such that for all $\beta \in[0,1]$, if $x$ and $y$ are comparable, then $A x$ and $A y, B x$ and $B y, A x$ and $B x$, $A y$ and $B y$ are comparable, respectively, and furthermore

$$
\begin{aligned}
\vee & \left(A x(A y)^{-1}, A y(A x)^{-1}\right)^{s \beta} \\
\lesssim & \vee\left(B x(B y)^{-1}, B y(B x)^{-1}\right)^{k \beta} \vee\left(A x(B x)^{-1}, B x(A x)^{-1}\right)^{h \beta} \\
& \vee\left(A y(B y)^{-1}, B y(A y)^{-1}\right)^{l \beta} .
\end{aligned}
$$

Then $A$ and $B$ have a common fixed point in $E$.

\section{Existence of a common solution for a system of integral equations}

The purpose of this section is to present an existence and uniqueness theorem for a solution of the following system of integral equations:

$$
\begin{aligned}
& x(t)=\int_{0}^{1} K_{1}(t, s, x(s)) d s+g(t), \quad t \in[0,1], \\
& x(t)=\int_{0}^{1} K_{2}(t, s, x(s)) d s+g(t), \quad t \in[0,1] .
\end{aligned}
$$

For $u, v \in \mathbb{R}$, if $u \leq v$ or $v \leq u$, we say that $u$ and $v$ are comparable.

We assume that

(i) $K_{1}$ or $K_{2}:[0,1] \times[0,1] \times \mathbb{R} \rightarrow \mathbb{R}$ is continuous, and $g:[0,1] \rightarrow \mathbb{R}$ is also continuous;

(ii) there exist $k \in(0,1)$ and a continuous function $\varphi:[0,1] \times[0,1] \rightarrow \mathbb{R}^{+}$such that if $u$ and $v$ are comparable, then $K_{1}(t, s, u)$ and $K_{2}(t, s, v)$ are comparable, furthermore

$$
\left|K_{1}(t, s, u)-K_{2}(t, s, v)\right| \leq k \varphi(t, s)|u-v|
$$

for $t, s \in[0,1]$ and $u, v \in \mathbb{R}$; 
(iii) $\sup _{t \in[0,1]} \int_{0}^{1} \varphi(t, s) d s \leq 1$;

(iv) there exists $x_{0} \in C[0,1]$ such that for any $t, s \in[0,1], x_{0}(t)$ and $\int_{0}^{1} K_{1}\left(t, s, x_{0}(s)\right) d s+g(t)$ are comparable.

Theorem 4.1 Under the assumptions (i)-(iv), then the integral equations have a unique common solution $x$ in $C[0,1]$.

Proof Let $E=C[0,1]$ be the set of real continuous functions defined on $[0,1]$ and $d: E \times$ $E \rightarrow[0,+\infty)$ be defined by $d(u, v)=\sup _{t \in[0,1]}|u(t)-v(t)|$. Then $(E, d)$ is a complete metric space. Set $P=\{x \in E: x(t) \geq 0$ for any $t \in[0,1]\}$ be a normal cone in $E$. The partial ordering in $E$ with respect to $P$ is given as follows:

$$
x \lesssim y \quad \Longleftrightarrow \quad x(t) \leq y(t) \quad \text { for any } t \in[0,1] .
$$

Also for every $x, y \in E$, there exists $w \in E$ such that $w$ is comparable with $x$ and $y$ [20].

Define $A, B: E \rightarrow E$ by

$$
\begin{aligned}
& A(x(t))=\int_{0}^{1} K_{1}(t, s, x(s)) d s+g(t), \quad t \in[0,1], \\
& B(x(t))=\int_{0}^{1} K_{2}(t, s, x(s)) d s+g(t), \quad t \in[0,1] .
\end{aligned}
$$

Obviously, the existence of a common solution for the integral equations is equivalent to the existence of a common fixed point of $A$ and $B$.

Now, from (ii), we have $A x$ and $B y$ are comparable if $x$ and $y$ are comparable for every $x, y \in E$. Also for each comparable $x, y \in E$, we obtain

$$
\begin{aligned}
|A(x(t))-B(y(t))| & =\left|\int_{0}^{1} K_{1}(t, s, x(s)) d s-\int_{0}^{1} K_{2}(t, s, y(s)) d s\right| \\
& \leq \int_{0}^{1}\left|K_{1}(t, s, x(s))-K_{2}(t, s, y(s))\right| d s \\
& \leq k \int_{0}^{1} \varphi(t, s) d s|x(t)-y(t)| \\
& \leq k\|x-y\|,
\end{aligned}
$$

which implies that $\|A x-B y\| \leq k\|x-y\|$.

Hence all of the conditions of Theorem 2.1 and Theorem 2.2 are satisfied, and so $A$ and $B$ have a unique common fixed point, which is a unique common solution of integral equations.

Notice that the example given above is in linear spaces. As to the noncommutative case, it is under consideration now. 


\section{Acknowledgments}

The authors thank referees for their careful reviewing of the manuscript and their remarkable suggestions which help to improve the quality of this paper. This work is supported financially by the NSFC $(10971011,11401022)$.

Received: 19 September 2014 Accepted: 17 May 2015 Published online: 04 June 2015

\section{References}

1. Jungck, G: Commuting mappings and fixed points. Am. Math. Mon. 83, 261-263 (1976)

2. Das, KM, Naik, KV: Common fixed point theorems for commuting maps on metric spaces. Proc. Am. Math. Soc. 77, 369-373 (1979)

3. Cirić, LB: A generalization of Banach's contraction principle. Proc. Am. Math. Soc. 45, 267-273 (1974)

4. Jungck, G: Compatible mappings and common fixed points. Int. J. Math. Math. Sci. 9, 771-779 (1986)

5. Berinde, $\mathrm{V}$ : A common fixed point theorem for compatible quasi contractive self mappings in metric spaces. Appl. Math. Comput. 213(2), 348-354 (2009)

6. Ćirić, LB, Ume, JS: Some common fixed point theorems for weakly compatible mappings. J. Math. Anal. Appl. 314(2), 488-499 (2006)

7. Ćirić, LB, Samet, B, Aydi, H, Vetro, C: Common fixed points of generalized contractions on partial metric spaces and an application. Appl. Math. Comput. 218(6), 2398-2406 (2011)

8. Abbas, M, Rhoades, BE: Common fixed point results for non-commuting mappings without continuity in generalized metric spaces. Appl. Math. Comput. 215, 262-269 (2009)

9. Aliouche, A: A common fixed point theorem for weakly compatible mappings in symmetric spaces satisfying a contractive condition of integral type. J. Math. Anal. Appl. 322, 796-802 (2006)

10. Shobkolaei, N, Sedghi, S, Roshan, JR, Altun, I: Common fixed point of mappings satisfying almost generalized $(S, T)$-contractive condition in partially ordered partial metric spaces. Appl. Math. Comput. 219, 443-452 (2012)

11. Huang, LG, Zhang, X: Cone metric spaces and fixed point theorems of contractive mappings. J. Math. Anal. Appl. 332(2), 1468-1476 (2007)

12. Abbas, M, Rhoades, BE, Nazir, T: Common fixed points for four maps in cone metric spaces. Appl. Math. Comput. 216, 80-86 (2010)

13. Cho, YJ, Saadati, R, Wang, SH: Common fixed point theorems on generalized distance in ordered cone metric spaces. Comput. Math. Appl. 61, 1254-1260 (2011)

14. Kadelburg, Z, Pavlović, M, Radenović, S: Common fixed point theorems for ordered contractions and quasicontractions in ordered cone metric spaces. Comput. Math. Appl. 59, 3148-3159 (2010)

15. Olaru, IM, Branga, A, Oprea, A: Common fixed point results in b-cone metric spaces over topological vector spaces. Gen. Math. 20(1), 57-67 (2012)

16. Ma, ZH, Jiang, LN, Sun, HK: C*-Algebra-valued metric spaces and related fixed point theorems. Fixed Point Theory Appl. 2014, Article ID 206 (2014)

17. Radenović, S, Rakočević, V, Resapour, S: Common fixed points for $(g, f)$ type maps in cone metric spaces. Appl. Math. Comput. 218, 480-491 (2011)

18. Song, GX, Sun, XY, Zhao, Y, Wang, GT: New common fixed point theorems for maps on cone metric spaces. Appl. Math. Lett. 32, 1033-1037 (2010)

19. Xin, QL, Jiang, LN: Fixed-point theorems for mappings satisfying the ordered contractive condition on noncommutative spaces. Fixed Point Theory Appl. 2014, Article ID 30 (2014)

20. O'Regan, D, Petruşel, A: Fixed point theorems for generalized contractions in ordered metric spaces. J. Math. Anal. Appl. 341(2), 1241-1252 (2008)

\section{Submit your manuscript to a SpringerOpen ${ }^{\ominus}$ journal and benefit from:}

- Convenient online submission

- Rigorous peer review

- Immediate publication on acceptance

- Open access: articles freely available online

- High visibility within the field

- Retaining the copyright to your article 